\title{
Distribution System Renewable Hosting Capacity Maximization with Second-Use Electric Vehicle Storage Using Critical Capacity Retention Calculation Model
}

\author{
Hang Jiang ${ }^{1}$, Li Tong ${ }^{1}$, Jian Zhao ${ }^{2, *}$, Qiushi Zhang ${ }^{2}$, Jingjing Zhao ${ }^{2}$ and Jinhui Zhou ${ }^{1}$ \\ 1 State Grid Zhejiang Electric Power Research Institute, Hangzhou 310006, China; jhshiep@163.com (H.J.); \\ tonyhust@126.com (L.T.); zhoujinhui_hz@163.com (J.Z.) \\ 2 Shanghai University of Electric Power, Shanghai 200090, China; zhangnicper@163.com (Q.Z.); \\ caojunjieee@foxmail.com (J.Z.) \\ * Correspondence: zhaojianee@foxmail.com; Tel.: +86-199-4560-8525
}

Received: 7 February 2020; Accepted: 24 March 2020; Published: 26 March 2020

\begin{abstract}
The continuous increase of the penetration of distributed generation in the distribution network poses more severe challenges for its full accommodation. In this context, an energy storage system can be installed to enhance the ability to accommodate renewable energy because of its high flexibility. However, the investment cost of brand new energy storage equipment is so high that the widespread application of storage system in the distribution system has been limited. This article proposes a method, which aimed at optimizing energy storage dispatching in a distribution network, and takes the use of second-use electric vehicles (EV) batteries into account. A calculation model of power battery second-use capacity was established, the upper and lower bounds of the initial capacity of second-use energy storage system (SUESS) can be determined after the reorganization of the retired batteries, and then a multi-objective optimal dispatching model for the distribution system with SUESS can be established with reference to the capacity attenuation model and the daily mileage statistics model of the electric vehicle. The case study results show that configured SUESS in the distribution network can not only meet the needs of the distribution network to fully accommodate distributed generation, but also delay the upgrade of the distribution network.
\end{abstract}

Keywords: second-use; electric vehicle batteries; battery capacity degradation; distribution network; renewable hosting capacity

\section{Introduction}

The rapid development of distribution network is inseparable from the important role that energy storage system plays in the solving problem that distributed power sources connect to grid. An energy storage system can be installed to effectively increase the system stability due to the large fluctuations and uncertainties of distributed power output. In addition, it can also effectively enhance the ability to accommodate renewable energy. At the user load side, the energy storage system can adjust the node voltage level, improve power quality, and optimize the power flow management of the distribution network $[1,2]$. The system also has some other applications that can be used, such as in electric vehicles, rail transit, and other industries.

Energy storage devices can be used as 'loads' and 'power sources'. Their dual attributes make energy storage devices a buffer source for power sources, which can ensure the stable and efficient output of distributed power sources, and serve as a backup 'power source' for power grids. At the same time, it can effectively guarantee the reliability of power supply and enhance the ability to 
accommodate renewable energy. Distributed power sources and storage system work together to solve the problem of higher power supply costs in remote areas. Energy storage devices greatly alleviate the shortage of power supply distance capability of large power grid systems, reduce system power supply costs, and reduce system failure rates. What is more, it is important that energy storage can be converted between two attributes, which can flexibly control its operation mode, and the response speed is very fast. In terms of the storage technology, it is widely used in the power system and can be used to connect new energy to the grid. It can be used to cut peaks and fill valleys, adjust frequency, provide backup, and improve the power grid's ability to absorb renewable energy [3].

In recent years, energy storage has received increasing attention, and the types of energy storage have been increasingly used, such as flywheel energy storage, compressed air energy storage, and electrochemical energy storage and electromagnetic energy storage. Nowadays, the number of new energy electric vehicles in China is increasing [4]. When the actual capacity of electric vehicle batteries is lower than a certain limit of rated capacity, it is difficult to meet the power needs of electric vehicles [5]. In terms of the retired batteries, the direct elimination of batteries will cause serious batteries recycling waste. Although decommissioned power batteries cannot meet their performance requirements in the field of electric vehicle applications, it can play its role on other occasions. The field of energy storage is one of the most important uses, such as for renewable energy power generation, peak-cutting and filling of smart grids, micro-grids, electric vehicle charging and replacing stations, and home backup power sources [5]. From the perspective of electric vehicles, the cost of power batteries accounts for almost one-third of the total cost of electric vehicles.

One of the most common functions of energy storage system is to improve the grid's ability to absorb renewable energy [6,7], but the widespread application of energy storage system in the power grid has been limited due to some factors. The biggest obstacle to distributed energy storage systems participating in grid operations is the high cost of batteries. Electric vehicles can participate in the optimal dispatch of the dispatch network [8,9] and the retired battery can be used to form an energy storage system. The contradiction caused by the high cost can be greatly alleviated if the cost of the battery can be reduced and the performance requirements in the energy storage field can be met.

The retention capacity of power battery will decrease as the number of cycle increases, and a battery residual capacity value model is established [10,11]. Reference [12] proposes a capacity decrease calculation model method that considers second-use electric vehicles batteries and used the low-cost electric vehicle retirement batteries to form an energy storage system. Reference [13] analyzes that, if the charge and discharge capacity of the retired power battery drops to $70-80 \%$ of the nominal capacity, the battery will be retired, and its battery will be recovered, disassembled, screened, and reorganized. A new energy storage system has been formed and applied to the optimal dispatching of the distribution network, which has greatly reduced the cost of energy storage system and enhance the ability to accommodate renewable energy. What is more, as a controllable load, the energy storage device can enable the distribution network to accommodate more renewable energy. Reference [14] proposes using energy storage devices to reduce the uncertainty of renewable energy sources and maximize the benefits of the distribution network by cutting peaks and filling valleys. Reference [15] proposes an optimal energy storage configuration scheme that solves the voltage limit of the distribution network, which reduces the working pressure of transformer taps and reduces the network losses. Reference [16] studies the ability of energy storage systems to alleviate line congestion.

Therefore, this paper calculates the capacity retention rate that is based on the number of charge and discharge cycles of the electric vehicle power battery, and determines the initial capacity limits of second-use energy storage system (SUESS) after the composition, and realizes the stepwise utilization of the electric vehicles power battery. Subsequently, the SUESS comprising the power battery is connected to the photovoltaic (PV) power in the distribution system, and the fluctuation of PV output is smoothed by charging and discharging of the SUESS. The main contributions of this paper can be summarized, as follows: 
(1) When compared with most of the previous works on distribution network operation with brand-new energy storage system, this paper proposes an optimal distribution system scheduling model that considers the integration of the second-use electric vehicle storage. Especially, this paper proposes a multi-objective distribution system scheduling optimization model for hosting capacity maximization of renewable energy with the coordination of charging and discharging strategy of the second-use electric vehicle storage.

(2) This paper innovatively proposes a critical capacity retention calculation model to model the dynamic capacity retention rate for second-use electric vehicle storage. Specifically, the number of battery cycles and energy storage cycle life loss are estimated while using this model. Electric vehicle travel behavior mileage model is established for calculating the initial capacity of second-use electric vehicle storage.

(3) Considering the inherent characteristics of the second-use electric vehicle storage, the paper proposes a SUESS embedded distribution system scheduling model that considers the dynamic updating of upper and lower bounds of the SUESS, as well as the dynamic updating of energy storage maintenance costs.

The rest of this paper is organized, as follows. In Section 2, a calculation model of power battery capacity retention rate in two life stages is established. The electric vehicles (EV) mileage model and capacity retention calculation model are established to calculate the initial capacity of the energy storage; cycle life loss model is established to dynamically update the energy storage capacity; the four-quadrant grid-connected operation model is established to explain the principle of energy storage participation in optimal scheduling. Subsequently, in Section 3, a day-ahead scheduling model with different objective functions is set up. Furthermore, simulation analysis is implemented on IEEE 37 test feeder in Section 4, and some useful conclusions are finally drawn in Section 5.

\section{Calculation Model of Power Battery Capacity Retention Rate in Two Life Stages}

Figure 1 depicts the simplified structure of distribution network with second-use electric vehicle energy storage system. In the dashed box, the second-use structure of an energy storage device that is composed of many electric vehicle retired batteries, replacing the new energy storage system. After decommissioned batteries are selected and reorganized, the retired batteries become a battery pack to participate in the optimal dispatch of the distribution network. There are two life stages of electric vehicles power battery from use to scrap, as can be seen from Figure 2. One is used for electric vehicles before decommissioning. The power battery for electric vehicles is applied to electric vehicles after system integration by the system integrator or a car manufacturer. This stage of battery application in electric vehicles can be called the first life of power batteries. Ideally, this stage can be 10-15 years. The other is to form an energy storage system that participates in the distribution network dispatch after decommissioning. This stage can be called the second life of power batteries. When the power battery capacity is less than $80 \%$ of the initial capacity, it is retired from the electric vehicle. Retired power batteries can be used in the some new scenarios, such as the field of energy storage, in order to achieve their level of utilization. The optimization work is in the second life stage of the power battery. At this stage, energy storage will cause its own capacity to decline, and the maintenance cost will then increase accordingly at this time, so a cost analysis model is added. 


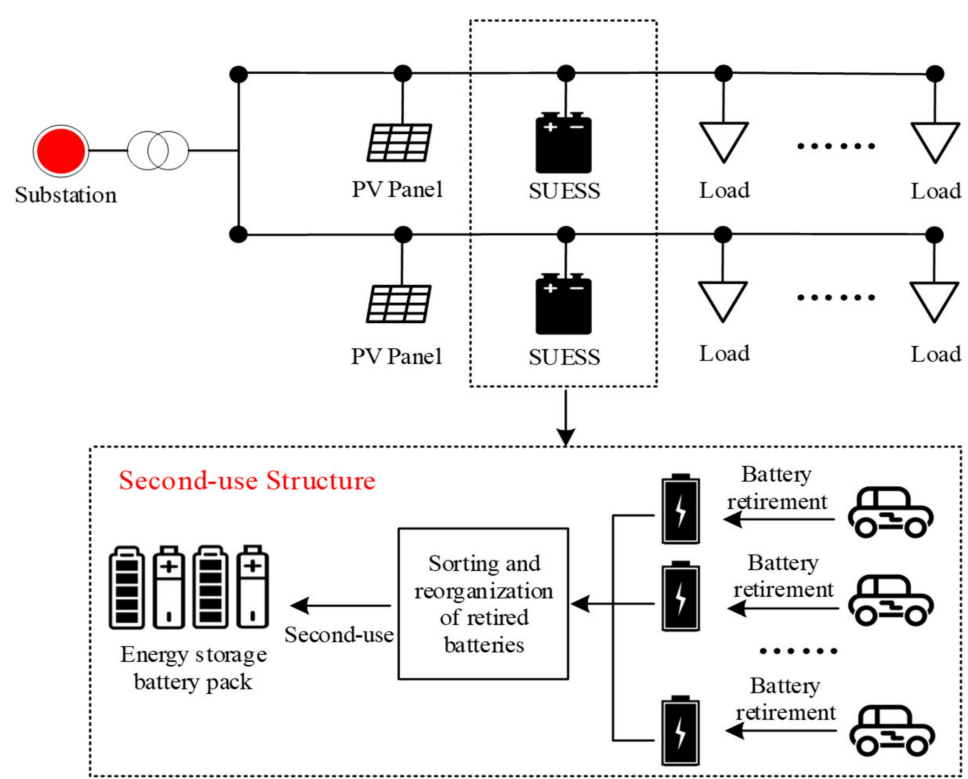

Figure 1. Simplified structure of distribution network with second-use electric vehicle energy storage system.

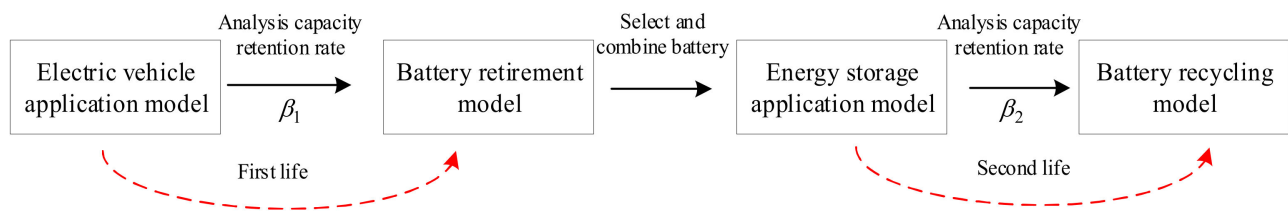

Figure 2. The two life stages of electric vehicles power battery.

\subsection{Calculation Model of Electric Vehicle Travel Mileage}

The annual cycle number of the power battery is closely related to the daily mileage of the electric vehicle, the power consumption per 100 kilometers, and the rated capacity of the electric vehicle power battery. Electric vehicles are still in the early stages of development and they lack statistical data on driving characteristics. According to the department of transportation's survey of household vehicles in the United States $[17,18]$, the daily mileage of vehicles is approximately a log-normal distribution, and Equation (1) shows its probability density function.

$$
f_{D}(x)=\frac{1}{\sqrt{2 \pi} \sigma_{D} x} \exp \left[-\frac{\left(\ln x-\mu_{D}\right)^{2}}{2 \sigma_{D}^{2}}\right]
$$

where $\mu_{D}=3.2, \sigma_{D}=0.88, \mathrm{D}$ is the daily mileage of the vehicle.

According to formula (1), it can deduce the expected value of the annual cycle number of power batteries.

$$
E(\bar{n})=\frac{B T_{\text {loss }}}{100} \cdot \frac{945.35 \cdot \exp \left(\mu_{D}+\frac{\sigma^{2} D}{2}\right)}{S_{b a t}}
$$

where $\mathrm{BT}_{\text {loss }}$ is the power consumption of electric vehicles per 100 kilometers, 945.35 represents 365 days multiplied by mileage conversion factor, and $\mathrm{S}_{\text {bat }}$ indicates the rated capacity of a single battery.

The annual cycle number of the electric vehicle battery is 127.4005 while assuming that the rated capacity of each power battery is $25 \mathrm{kWh}$, which can be derived from formula (2). Within the specified system life, the above-mentioned sampling process is repeated, and the second-use capacity of the power battery in the following year is finally obtained. 
For each power battery, the number of cycles is calculated based on a certain probability distribution, and accumulate the scatter value to the number of accumulation cycles of the battery, as shown in Equation (3):

$$
\left\{\begin{array}{lrl}
\eta_{i}^{\text {sum }}=\phi_{i,} & i=1 \\
\eta_{i}^{\text {sum }}=\eta_{i-1}^{\text {sum }}+\phi_{i}, & i=2,3, \cdots, m
\end{array}\right.
$$

where $\varphi_{i}$ is the cycle number of the i year obtained by random sampling of the power battery, the value of $\phi_{i}$ is equal to $\mathrm{E}(\bar{n}) ; \eta_{i}^{\text {sum }}$ is the cumulative cycle number of the power battery in the i year.

The battery can continue to be used if the cumulative number of cycles of the power battery is less than a specified value; if the cumulative number of cycles is larger than or equal to the specified value, the battery needs to be downgraded, and it is used to become the part of SUESS. The corresponding power battery is replaced with a new battery; the cumulative cycle number $\eta_{i}^{\text {sum }}$ of the battery is corrected to zero. In this article, it can be analyzed that, when the cumulative number of cycles reached 1400 , the critical capacity retention rate reached $80 \%$, and the battery is retired. The following process is shown in Equation (4)

$$
\eta_{i}^{\text {sum }}= \begin{cases}\eta_{i}^{\text {sum }}, & \eta_{i}^{\text {sum }}<\eta^{\text {lim }} \\ 0, & \eta_{i}^{\text {sum }} \geq \eta^{\lim }\end{cases}
$$

where $\eta^{\text {lim }}$ is the specified value of the number of cycles in order to determine whether the power battery is degraded, being referred to as the number of critical cycles, which can be judged based on the capacity degradation characteristics and the critical capacity retention rate.

\subsection{Electric Vehicle Power Battery Critical Capacity Retention Calculation Model}

The capacity of the electric vehicle power battery that is used for annual degradation is not easy to determine, so it is necessary to know the real capacity degradation at the beginning of second life. When considering the capacity degradation characteristics of the power battery and the electric vehicle driving characteristics, based on travel mileage model of electric vehicle in Section 2.1, the calculation model of second-use capacity is established to obtain the capacity degradation with cycling.

In this article, the capacity retention rate $\beta$ represents the ratio of the real capacity to the rated capacity, the capacity 'dividing point' that determines the second use of power battery is called the critical capacity retention rate, which is recorded as $\beta_{\text {lim }}$ (usually about $80 \%$ ).

Due to the increase of cycles, the real available capacity of the power battery gradually decreases, and the capacity retention rate $\beta$ with the number of cycles $\eta$ basically conforms to the mathematical relationship of the function [19]:

$$
\beta_{1}=\beta_{0}-a \eta+b \eta^{2}-c \eta^{3}
$$

where $\beta_{1}$ is the capacity retention rate of the power battery; $\eta$ is the number of cycles; $\beta_{0}, a, b$, and $c$ are the parameters to be determined. The four parameters are taken $100.37,0.018,3 \times 10^{-6}$ and $4 \times$ $10^{-10}$. Therefore, the relationship between $\alpha$ and $\mathrm{n}$ can be expressed as:

$$
\beta_{1}=100.37-0.018 \eta+3 * 10^{-6} \eta^{2}-4 * 10^{-10} \eta^{3}
$$

In the first life stage, the purpose of calculating the capacity retention rate is to determine the capacity when the battery is decommissioned, thus determining the initial capacity of the SUESS.

\subsection{Energy Storage Cycle Life Loss Model}

Battery cycle life expectancy will be reduced by factors, such as operating temperature, peak current, and number of charge-discharge cycles. The depth of discharge (DOD) can also directly affect its cycle life, which is recorded as D. This article uses the rain current counting method to calculate the discharge depth of the energy storage battery. Subsequently, the storage cycle life at this discharge depth can be calculated. 
Among various battery energy storage materials, because of the advantage, such as high charge efficiency, long cycle life, and both high specific energy and high specific power, lithium battery is widespread used, so it is considered to be the most promising battery materials in storage for power systems. A fourth-order polynomial function fits the curve, and the function relationship is as follows:

$$
K(D)=a D^{4}-b D^{3}+c D^{2}-d D+e
$$

where $K(D)$ is the storage cycle life at this discharge depth. The five parameters are taken 7,1470,170,100, $146,400,56,500$, and 12,230 . Therefore, the relationship between $K(D)$ and $D$ can be expressed, as:

$$
K(D)=71470 D^{4}-170100 D^{3}+146400 D^{2}-56500 D+12230
$$

The energy storage operating conditions can be regarded as a series of linear combinations of charge and discharge cycles with different discharge depths. The operating conditions are decomposed into multiple conditions with different discharge depths. The energy storage cycle life under each condition after decomposition is calculated. The linear storage of the energy storage cycle life loss is directly obtained according to the cycle times of the energy storage under each corresponding working condition. The cycle life loss of energy storage during operation can be calculated, as follows:

$$
L=\sum_{D=0}^{1} \frac{m}{K(D)}
$$

where $m$ is the number of energy storage cycles at the discharge depth.

The number of cycles and the corresponding discharge depth of the stored energy can be counted by the rain current counting method, and the cycle life loss of the stored energy during the operation can then be obtained by formulas (8) and (9). When $L=1$, the energy storage life is considered to be exhausted, and the energy storage needs to be replaced.

The China Electric Power Research Institute performed secondary cycle tests on batteries retired from electric vehicles. The test results show that the cycle life of the decommissioned power battery when it is in the second life stage can be roughly estimated [20].

$$
\beta_{2}=-2.6043 \times 10^{-5} n+0.8347
$$

where $n$ is the number of cycles of the SUESS; $\beta_{2}$ is the capacity retention rate of the storage system.

\subsection{Storage System Power Four-Quadrant Grid-Connected Operation}

The SUESS includes battery energy storage and energy storage converter PCS. Figure 3 shows the main circuit topology. The battery energy storage device releases or absorbs electric energy, and PCS is a self-commutated three-phase full-bridge inverter and capacitor $C$ provides DC voltage support for the inverter. $L_{a}, L_{b}$, and $L_{c}$ are the equivalent inductance at the BESS outlet side that change the phase between the output voltage and current. $U_{s a}, U_{s b}$, and $U_{s c}$ are the three-phase voltages of the distribution network. By controlling the on and off of power electronic devices, the output voltage can be changed in size and phase to achieve independent and fast adjustment of active and reactive power.

The SUESS controller is generally divided into dual-loop control. The outer-loop controller calculates the control demand that is required by the distribution network that is based on the actual electrical data that were collected on the scene, and then decouples the active and reactive power to give the final decoupled power demand instruction. It is sent to the inner-loop controller, and the inner-loop controller calculates the trigger signal of the device switch according to the obtained instruction to realize the four-quadrant real-time decision making of the system output power. 


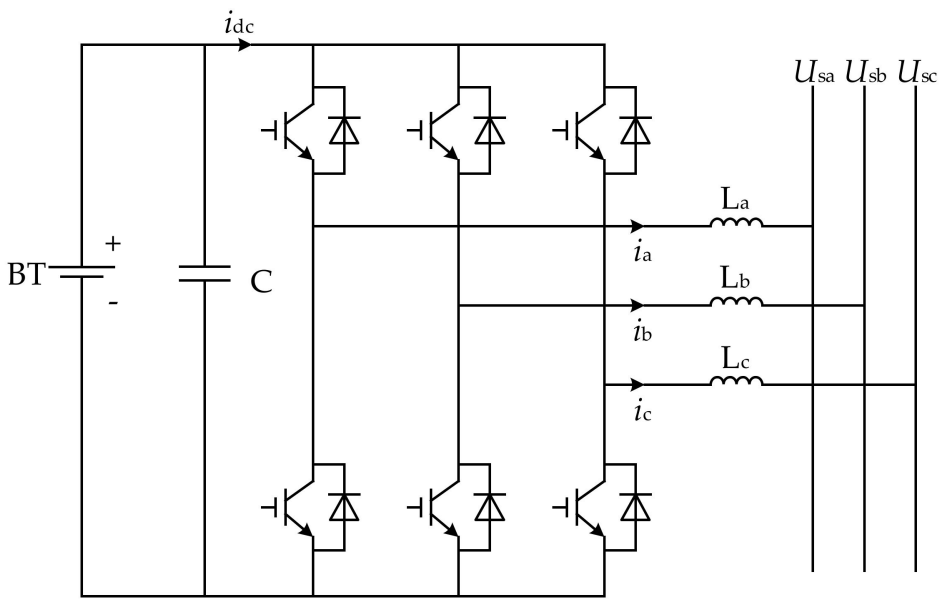

Figure 3. Main circuit structure of second-use energy storage system (SUESS).

In the case of ignoring the equivalent impedance power loss at no load of SUESS, the formula of power exchange between the distribution network and BESS is approximately:

$$
P^{Q U}+Q^{Q U}=\left(\frac{U_{S} U_{t}}{Z_{L}}\right)^{2}
$$

where $U_{s}$ is the amplitude of the voltage; $U_{t}$ is the amplitude of the AC voltage of the SUESS after inverter inversion; and, $Z_{L}$ is the network impedance. With the network voltage $U_{s}$ and impedance $Z_{L}$ determined, the output power of active power $P_{0}$ and reactive power $Q_{0}$ depends on the phase and size of the SUESS output voltage $U_{t}$. A circle can describe Formula (11). The size of $U_{t}$ determines the radius of the circle, and the phase of $U_{t}$ determines which position on the circle the SUESS operating point is on; where, $P Q U Q^{Q U}$ are used to estimate the quadratic terms of $P$ and $Q$.

The figure shows the generation and adjustment of reactive power. The battery generates DC power, and, by controlling the frequency of the on-off signal, adjusting the duty cycle to output AC power. The phase between the output voltage and current changes due to the addition of inductive elements, thereby generating reactive power. Secondly, adjust the reactive power output of the energy storage battery by changing the phase of $U_{t}$ in formula (11).

This paper proposes piecewise linearization Distflow, which is implemented by linearizing the quadratic terms of active power and reactive power, so that the loss term can be maintained [21]. This paper uses formulas (12-14) for the linearization for the convenience of simulation. The quadratic terms of parameters, such as power $\mathrm{P}, \mathrm{Q}$, and $\mathrm{U}$, can be implemented with the following constraints:

$$
\begin{aligned}
& P_{j t}^{Q U} \geq K_{\gamma j t}^{A P} P_{j t}+B_{\gamma j t}^{A P}, \forall j, t, \forall \gamma \in \Gamma^{A P} \\
& Q_{j t}^{Q U} \geq K_{\gamma j t}^{R P} Q_{j t}+B_{\gamma j t}^{R P}, \forall j, t, \forall \gamma \in \Gamma^{R P} \\
& U_{s t}^{Q U} \geq K_{\gamma s t}^{U P} U_{s t}+B_{\gamma s t}^{U P}, \forall t, \forall \gamma \in \Gamma^{U P}
\end{aligned}
$$

where $P_{j t}^{Q U} Q_{j t}^{Q U}$ are used to estimate the quadratic terms of $P_{j t}$ and $Q_{j t}, K_{\gamma j t}^{A P} K_{\gamma j t}^{R P}$ and $B_{\gamma j t}^{A P} B_{\gamma j t}^{R P}$ are the constant coefficients of linear approximation. For the sake of description, in subsequent constraints, this paper still use $P_{j t}$ instead of $P_{j t}^{Q U}$, use $Q_{j t}$ instead of $Q_{j t}^{Q U}$, and use $V_{t}$ instead of $U_{Q U} s t$.

When the outer loop controller in the PCS detects the active and reactive power required by the distribution network, the inner loop controller controls the SUESS active and reactive power output to be larger than zero. At this time, the SUESS releases the active and reactive power to the grid, and the state of battery is discharging, external reactive power compensation is performed at the same time, which corresponds to the SUESS working mode in the first quadrant; when the outer loop controller 
detects active and reactive power surplus in the distribution network, the inner loop controller controls the active and reactive power output to be less than zero. At this time, the power grid sends active and reactive power to the SUESS, the battery is in a charging state, and the capacitor absorbs reactive power from the distribution system, which corresponds to the SUESS operating mode in the third quadrant. Similarly, the SUESS can also work in the second, fourth quadrants, or coordinates where it can flexibly control its charge and discharge status according to actual outside needs. The operation strategy of the SUESS is derived through economic optimization based on the typical daily load. It can be maximized renewable hosting capacity under the condition of meeting all of the limits set by the distribution network.

\section{Multi-Objective Optimal Dispatching Model}

As the penetration rate of renewable energy access is getting higher and higher, improving the carrying capacity of renewable energy is very important for the economic operation of the distribution network [22-24]. This paper uses coordinated control between second-use energy storage and photovoltaic power generation in order to achieve optimal dispatch operation of the distribution network. The multi-objective optimal scheduling model that is proposed in this paper considers two sources of uncertainty in the distribution network: photovoltaic power output and load demand. In order to deal with these two uncertainties, a large number of historical data in [25] are used, and a set of representative uncertainty sets is then selected through the perfect reverse reduction algorithm that was proposed in [26] and those can be input to the day-ahead stage. In this paper, rolling scheduling optimization is used to input the load data in real time, which has calculated the impact of uncertainty. This article introduces SUESS to replace new and high-cost energy storage systems, and it calculates the SUESS maintenance costs that are involved in the optimal operation of the distribution network based on the capacity retention rate of the second-use storage, so that the distribution network achieves optimal economics.

\subsection{Objective Function}

The objective function considers three aspects in order to reduce the operating costs as much as possible under the conditions of system operation: the maximum photovoltaic hosting capacity, the minimum energy storage investment cost and the penalty costs for voltage and capacity violations:

$$
\operatorname{Min} f=\left(f_{1}+f_{2}+f_{3}\right)
$$

3.1.1. Minimum PV Curtailment

$$
f_{1}=\sum_{m \in \Omega^{p v}(j)} E_{m}-P_{m}
$$

where $\Omega^{p v}(j)$ is the indices set of PV nodes, $\mathrm{E}_{\mathrm{m}}$ and $\mathrm{P}_{\mathrm{m}}$ are, respectively, the total available and actual output power.

\subsubsection{Minimum Energy Storage Cost}

$$
f_{2}=K_{E} E_{0}^{\text {suess }}+K_{p} P_{0}^{\text {suess }}
$$

where $\mathrm{K}_{E}$ represents unit capacity maintenance cost, which is affected by the capacity retention rate of the SUESS; $\mathrm{K}_{p}$ represents energy storage converter PCS operation and maintenance unit price; and, $E_{0}^{\text {suess }}$ and $P_{0}^{\text {suess }}$ are the rated capacity and rated power of the SUESS.

The relationship between the unit operation and maintenance cost of the SUESS with initial capacity retention rate of the storage can be fitted with an exponential function, as shown in Equation (18). 
The function of this formula is to dynamically update the energy storage maintenance cost based on the accumulated number of cycles of the energy storage system. Figure 4 gives a more detailed explanation.

$$
K_{E}=0.05165 \times \beta^{-6}
$$

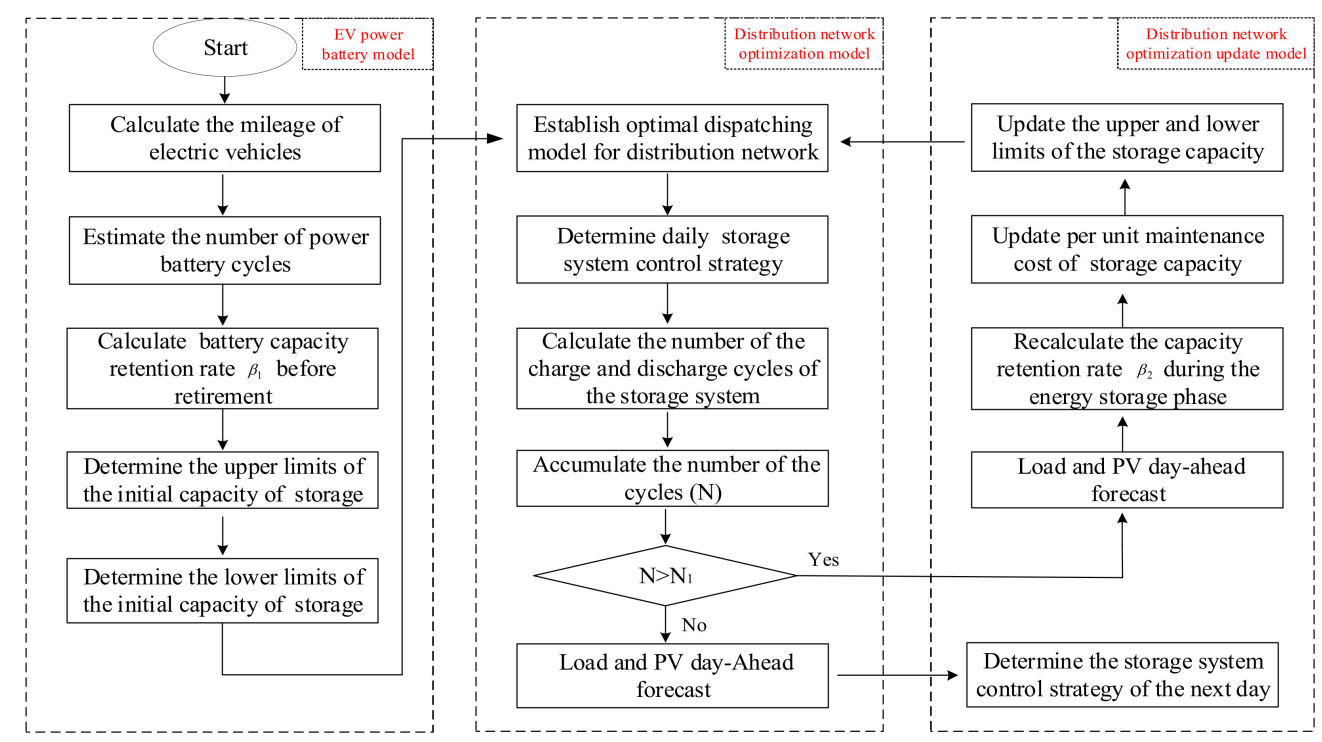

Figure 4. The framework of SUESS embedded distribution system scheduling model.

\subsubsection{Minimum Penalty Cost}

$$
f_{3}=\sum_{t \in T} \sum_{j \in J} c^{\text {penalty }}\left(s_{j t}^{v, l b}+s_{j t}^{v, u b}\right)
$$

where $c^{\text {penalty }}$ is a very large positive number, which is used to avoid node voltage amplitude exceeding limits, $s_{j t}^{v, l b}$ and $s_{j t}^{v, u b}$ are included in (23) and (24) to relax bus voltage constraints, respectively.

\subsection{Constraints}

First, the power flow balance constraint is given to ensure the safe operation of the system, and then the operational constraints of the SUESS are given.

\subsubsection{Power Flow Constraints}

$$
\begin{gathered}
P_{j t}=\sum_{i \in \varphi(j)} P_{i t}+p_{j t}-\sum_{m \in \Omega^{p v}(j)} P_{m}^{p v}-\sum_{l \in \Omega^{\text {suess }}(j)} P_{l t}^{\text {suess }}, \forall j, t \\
Q_{j t}=\sum_{i \in \varphi(j)} Q_{i t}+q_{j t}+\sum_{b \in \Omega^{s v c}(j)} Q_{b t}^{s v c}, \forall j, t
\end{gathered}
$$

where $j$ represents a node $j$ in the distribution network, $t$ represents the $t$ time phase, $i$ represents the sub-node of the node $j, m$ represents the PV installation location, $b$ represents the SVC installation location, and $l$ represents the SUESS installation location; $\varphi(j)$ indicates the sub-node set of node $j$, $\Omega^{p v}(j)$ indicates the sub-node set of node $j$ with PV, $\Omega^{s v c}(j)$ indicates the sub node set of node $j$ with SVC, and $\Omega^{\text {suess }}(j)$ indicates the sub node set of node $j$ that is equipped with the SUESS.

Equations (20) and (21) describe the active and reactive power balances at each node, respectively. $P_{j t}$ and $Q_{j t}$ represent the active and reactive power of node $\mathrm{j}$ at time $\mathrm{t}, p_{j t}$ and $q_{j t}$ represent the active and reactive load of node $\mathrm{j}$ at time $\mathrm{t}, P_{m}^{p v}, P_{l t}^{\text {suess }}$, and $Q_{b t}^{s v c}$ are the output of PV, SUESS, and SVC at time t. 


$$
\begin{gathered}
V_{j t}=V_{i t}+\frac{r_{i j} P_{i t}+x_{i j} Q_{i t}}{V_{0}}, \forall i \in \varphi(j), \forall j, t \\
V_{j t}+s_{j t}^{v, l b} \geq V^{\min }, \forall j, t \\
V_{j t}+s_{j t}^{v, u b} \leq V^{\max }, \forall j, t
\end{gathered}
$$

Equation (22) describes the voltage relationship between two adjacent nodes. $r_{i j}$ and $x_{i j}$ respectively represent the line resistance and the reactance between node $i$ and $j, V_{j t}$ represents the voltage of node $j$ at the time $t$.

\subsubsection{Energy Storage System Operation Constraints}

$$
E_{l t}^{\text {suess }}=E_{l t-1}^{\text {suess }}+\eta^{c h} P_{l t}^{c h}-\eta^{d c h} P_{l t}^{d c h}, \forall l, t
$$

where $\eta^{c h}$ and $\eta^{d c h}$ respectively represent the charging and discharging efficiency; $P_{l t}^{c h}$ and $P_{l t}^{d c h}$ respectively represent the charging and discharging power at time $t ; E_{l t}^{\text {suess }}$ represents the SUESS capacity at time t. Equation (25) describes the state of charge of the SUESS at each time period.

$$
\begin{gathered}
E^{\text {suess,min }} \leq E_{l t}^{\text {suess }} \leq E^{\text {suess,max }}, \forall l, t \\
E^{\text {suess,min }}=\beta E_{0}^{\text {suess, } \min } \\
E^{\text {suess, } \text { max }}=\beta E_{0}{ }^{\text {suess, } \max }
\end{gathered}
$$

where $E_{0}^{\text {suess }}$ represents the initial total capacity of the original brand new battery pack; $\beta$ is the capacity retention rate of the SUESS. $E^{\text {suess, } \min }$ and $E^{\text {suess, } m a x}$ represent the minimum and maximum values of the energy storage capacity, respectively. Equation (26) limits the overcharge and over-discharge of the SUESS.

$$
\begin{gathered}
0 \leq P_{l t}^{c h} \leq u_{l t}^{c h} P^{c h, \max }, u_{l t}^{c h} \in\{0,1\}, \forall l, t \\
0 \leq P_{l t}^{d c h} \leq u_{l t}^{d c h} P^{d c h, \max }, u_{l t}^{d c h} \in\{0,1\}, \forall l, t \\
u_{l t}^{c h}+u_{l t}^{d c h}=1, \forall l, t
\end{gathered}
$$

where $P^{c h, m a x}$ and $P^{d c h, m a x}$ represent the maximum charge and discharge power of the energy storage system, respectively; $u_{l t}^{\text {ch }}$ (takes 1 when charging) and $u_{l t}^{\text {dch }}$ (takes 1 when discharging) are $0-1$ variables. Equations (29) and (30), respectively, represent the charge and discharge power allowed by the limit of SUESS. Equation (31) limits the simultaneous charging and discharging of the energy storage system.

After the power battery is retired, its capacity retention rate is estimated in order to determine the upper and lower limits of the capacity of the SUESS. The capacity retention rate is continuously decreasing due to the increase in the number of alternate cycles of the battery during the phase of energy storage participating in optimal scheduling. At this time, the energy storage capacity for peak cutting and valley filling will decrease, and the energy storage needs to be recalculated. The capacity maintenance rate can be updated, and its unit maintenance cost is updated at the same time, these parameters are used as initial values for the next day, and then re-executed for optimal scheduling.

As the energy storage participates in the optimized operation of the distribution network, the number of cycles gradually increases, and the capacity retention rate then decreases, the maintenance cost becomes higher and higher. During the optimization process, the number of cycles will always increase. After completing the scheduling plan, that is, the formulation of the energy storage control strategy, and then accumulate the number of battery cycles to calculate the capacity retention rate. When the capacity retention rate is reduced by $1 \%$, the maintenance cost in the objective function 
changes its value, and then participate in the new optimal scheduling to obtain a coordinated energy storage control strategy.

- scenario one: when the battery capacity retention rate $\beta$ is 0.8 , the unit capacity maintenance cost $K_{E}$ is $0.197(\$ / \mathrm{kWh})$. The update process is as follows:

Step1: Forecast the PV total available output power $E_{m}^{p v}$ and the load data $p_{j t}$ for the next day; Step 2: Update formula (13) according to the unit maintenance cost $K_{E}$ in this scenario; Step3: Calculate the upper and lower limits of the energy storage capacity based on the equation $E^{e s s, \min }=\beta E_{0}^{e s s, \min }$ and $E^{e s s, \max }=\beta E_{0}^{e s s, \max }$.

- scenario two: when the battery capacity retention rate $\beta$ battery capacity is 0.79 , the unit capacity maintenance cost $K_{E}$ is $0.212(\$ / \mathrm{kWh})$. Update process is the same as above.

- scenario three: when the battery capacity retention rate $\beta$ battery capacity is 0.78 , the unit capacity maintenance cost $K_{E}$ is $0.229(\$ / \mathrm{kWh})$. Update process is the same as above. ......

- $\quad$ scenario $\mathrm{n}$ : when the battery capacity retention rate $\beta$ battery capacity is $0.8-0.1 *(n-1)$, the unit capacity maintenance cost $K_{E}=0.05165 \times \beta^{-6}(\$ / \mathrm{kWh})$. Update process is the same as above.

\section{Case Study}

\subsection{Case Description}

This article uses the modified IEEE 37 system to test the optimal dispatching model [27]. Figure 5 shows the modified 37-nodes system. The algorithm is implemented on MATLAB based on CPLEX. Photovoltaic and energy storage devices are installed at the 19 nodes that are shown in the Figure 5. The capacity of distributed photovoltaic is $100 \mathrm{~kW}$ and the capacity of the SUESS is $50 \mathrm{~kW} / 200 \mathrm{kWh}$. All of the energy storage systems of each node that has been installed are referred to as ESS. $\eta^{\text {ch }}$ and $\eta^{d c h}$ are both 0.9 ; both $P^{c h, m a x}$ and $P^{d c h, m a x}$ are $4 \mathrm{~kW}$ and many other parameters are dynamically changing quantities.

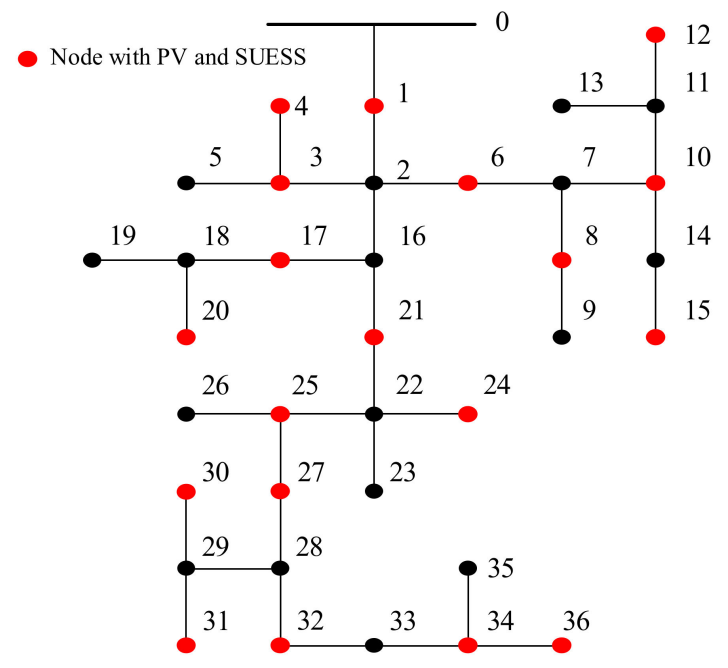

Figure 5. Modified IEEE 37 test system.

\subsection{Results and Analysis}

\subsubsection{Results of Two-Stage Capacity Decay of Retired Batteries}

Figure 6 shows the relationship between the battery capacity and the number of cycles in the life cycle of an electric vehicle. It can be seen that, when the battery is retired, that is, when the capacity retention rate drops to $80 \%$, the number of power battery cycles is close to 1400 ; Figure 7 shows that, 
with the continuous use of retired power batteries, the capacity of the battery is gradually reduced, according to the capacity decay formula. It is assumed that the initial available battery capacity is $83.47 \%$, and the available battery capacity is reduced to $72.06 \%$ after the third year. The decline of battery capacity will reduce the cycle interval of energy storage step utilization, and the energy storage capacity that is used for peak clipping and valley filling will decrease, which will affect the efficiency of step utilization. Once the energy storage system is unable to meet the externally required active and reactive power due to the reduced capacity, it might cause hazards, such as voltage rise. In addition, when maintenance costs are too high, the old energy storage system needs to be replaced in a timely manner.

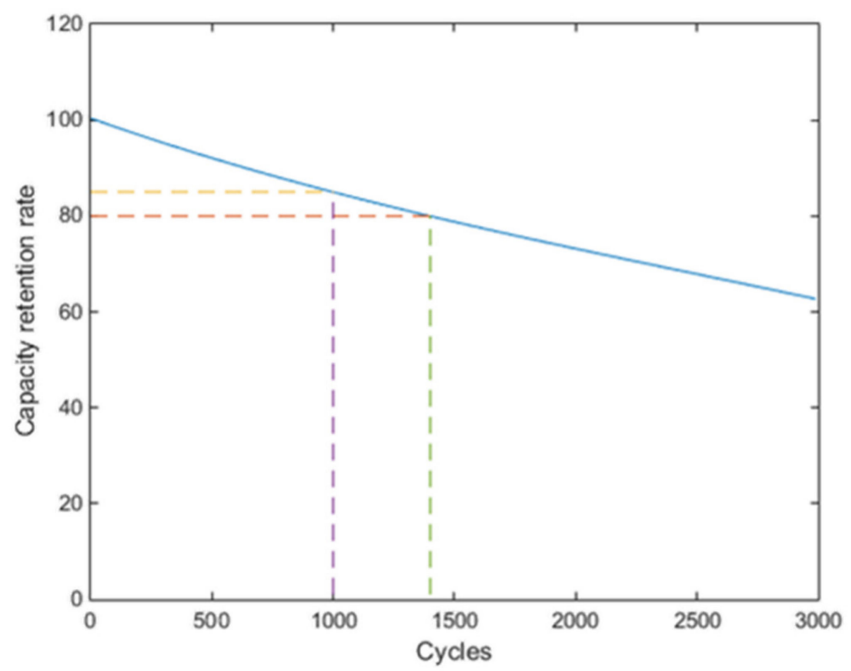

Figure 6. The relationship between the number of cycles and capacity retention rate.

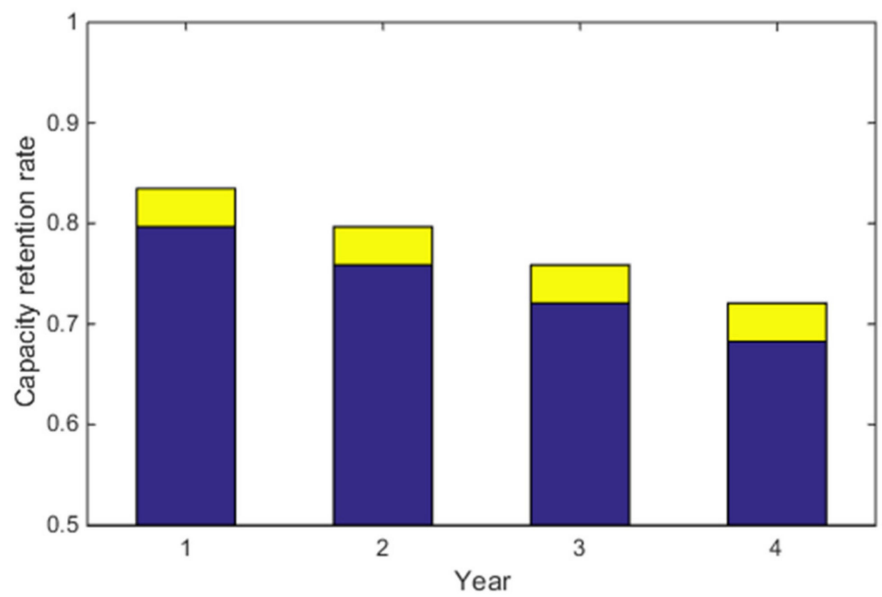

Figure 7. Storage battery capacity decays year by year.

\subsubsection{Load PV and Energy Storage System Output Power}

The results of the optimized operation of the distribution network with energy storage photovoltaic are as follows:

It can be seen from the analysis results illustrated in Figures 8 and 9 that the PV output from 12:00 to 15:00 is larger than the value of load demand. At this time, the SUESS is charged to absorb the excess PV output power, and the state of charge (SOC) gradually increases. At the night peak, the energy storage system emits power, and the SOC gradually decreases. It is charged when the load level is low in the early morning, and the SOC gradually increases, and it emits power at the early peak. After the determination of the energy storage location, in Figures 10 and 11, it can be found that after the SUESS 
installation, the voltage deviation level of the node is reduced, which is beneficial to the safe operation of the distribution network, according to the optimization results. The total voltage deviation during a dispatch period, respectively, has decreased by 0.084 (p.u.) and 0.079 (p.u.), which indicates that the participation of the energy storage system significantly improves the problem of voltage deviation and ensures the safety of distribution network operation. The above analysis shows that SUESS composed of retired batteries can also meet the role of brand new energy storage, which verifies the rationality of the second-use energy storage batteries participating in the optimal dispatching of the distribution network, and it proves the residual value of the retired batteries.

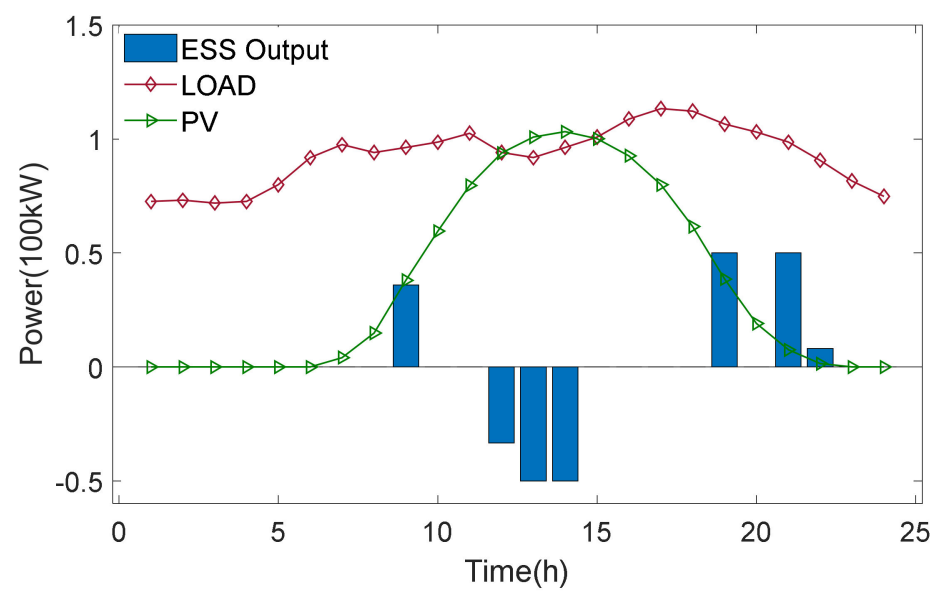

Figure 8. The fourth node load, photovoltaic (PV), and storage output curve.

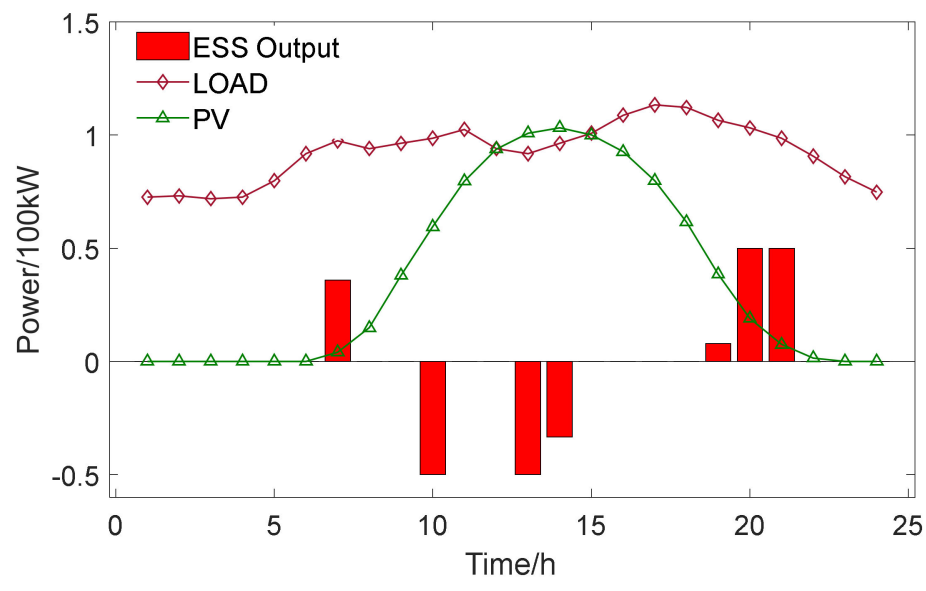

Figure 9. The tenth node load, PV, and storage output curve.

In actual situations, the dispatching period of the power grid is generally $15 \mathrm{~min}$. or $30 \mathrm{~min}$. Since the actual data (PV data, load data, etc.) used in this paper are hourly data, the size of the energy storage charge and discharge in the optimization variables is also an hourly variable, and the figures show step changes in battery SOC. Figures 12 and 13 show the capacity rate status of SUESS in a day. It can be seen that the SUESS composed of power batteries not only improves the system's ability to absorb PV and reduce the peak-to-valley difference, but also the total investment cost of the energy storage system composed of power batteries is lower than that of the new energy storage system, according to the optimization results above.

Figure 14 shows the network loss of the system under the two cases. The network loss that adds SUESS with power four-quadrant operation is reduced by 0.1958 (p.u.) than traditional energy storage. The network loss of the system under the two cases is almost the same due to the small impact of PV output from 0:00 to 7:00. The SUESS output of the four-quadrant power can be regulated by reactive power to avoid unnecessary power loss. From the figure, it can be observed that the network loss 
has greatly reduced. The network loss is directly dependent on the line current amplitude. The line current amplitude is positively related to the active and reactive power flowing through the line and negatively related to the voltage of the first node. Only the active power portion of the line can be reduced if only the active power adjustment is performed. Therefore, the total network loss of pure active power adjustment results will naturally be relatively high.

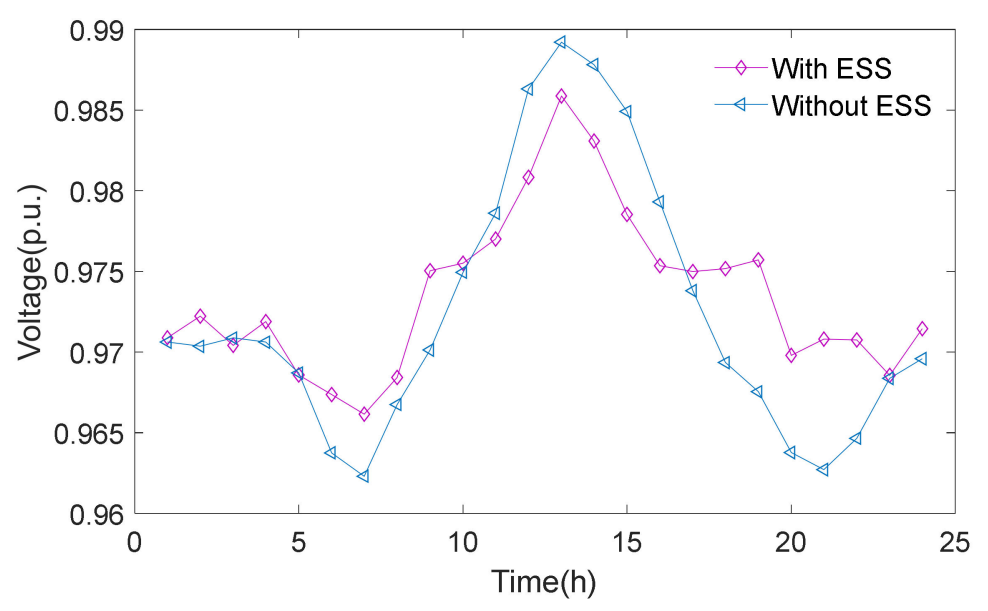

Figure 10. The fourth node voltage.

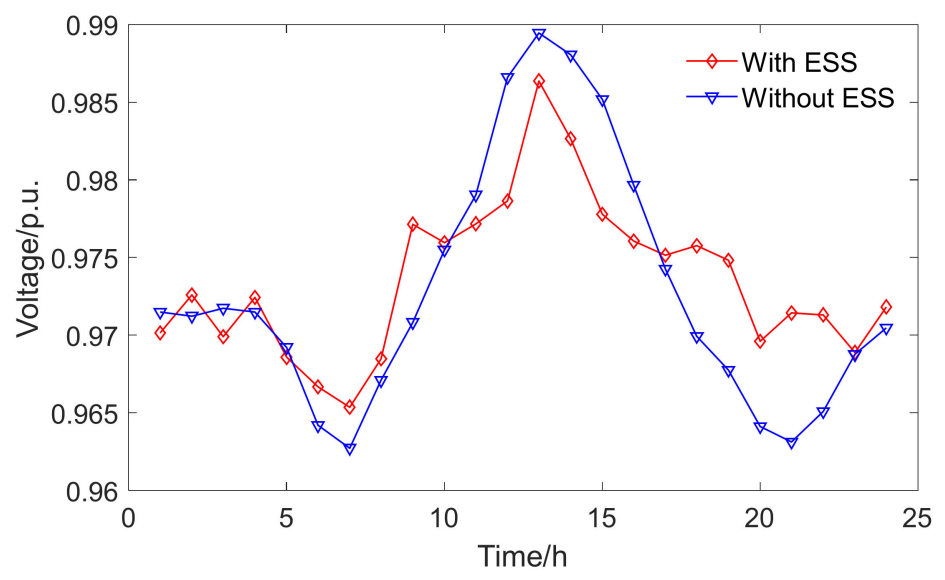

Figure 11. The tenth node voltage.

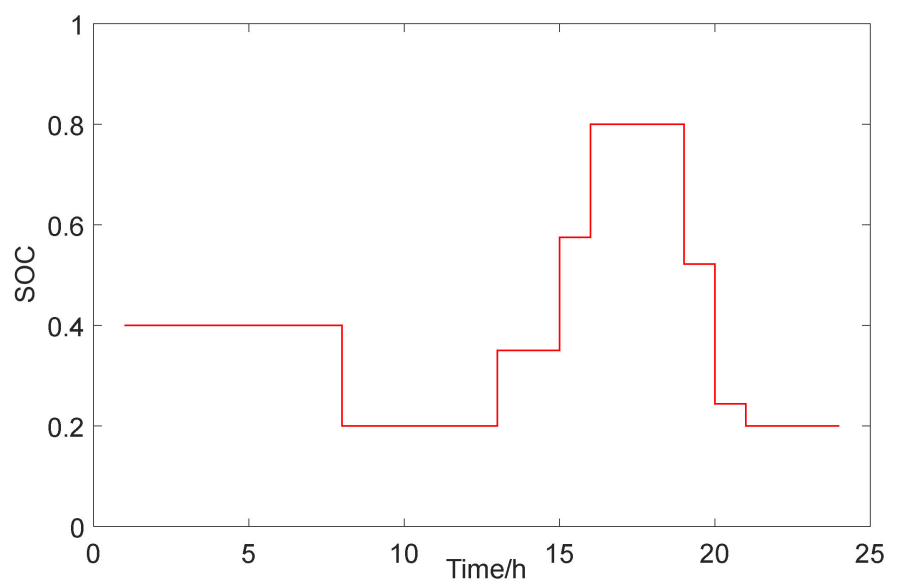

Figure 12. State of charge (SOC) of storage 4. 


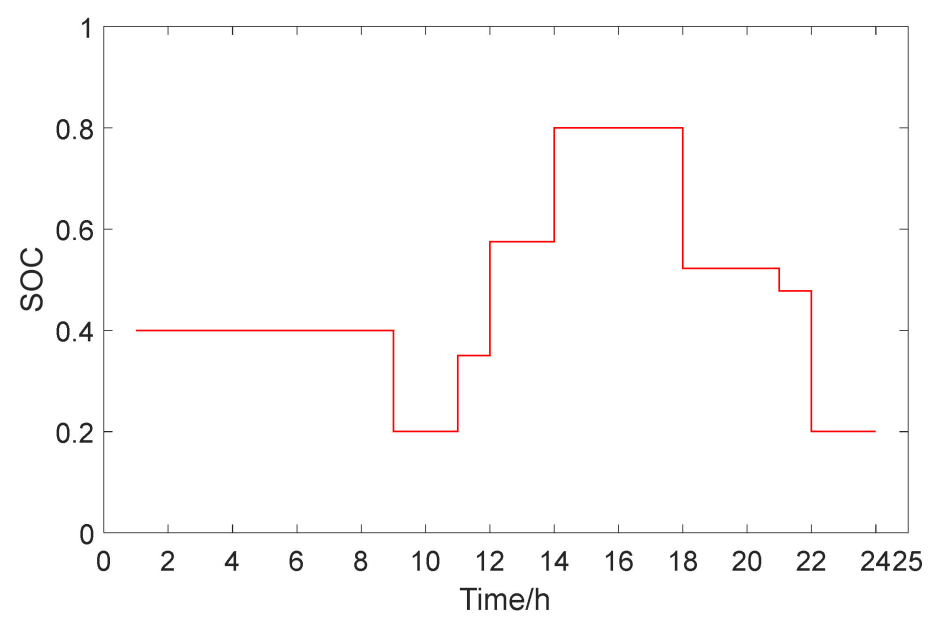

Figure 13. SOC of storage 1.

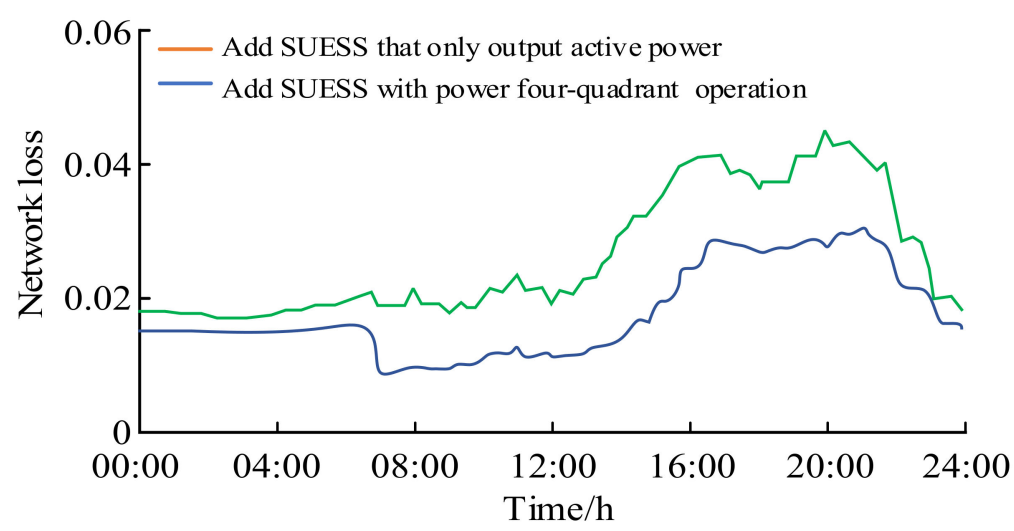

Figure 14. Two cases of the distribution network loss.

Figure 15 shows the maintenance cost analysis of SUESS. Before the attention rate changes by $1 \%$, the cumulative optimal cost of the simulation is $68204 \$$, and the cost of purchasing a brand new energy storage to participate in the operation is $88837.5 \$$, it can be calculated that the cost of using SUESS in this model is less than the brand new energy storage system by $20633.5 \$$. Accordingly, it can be seen that the use of the energy storage cost in stages is economical.

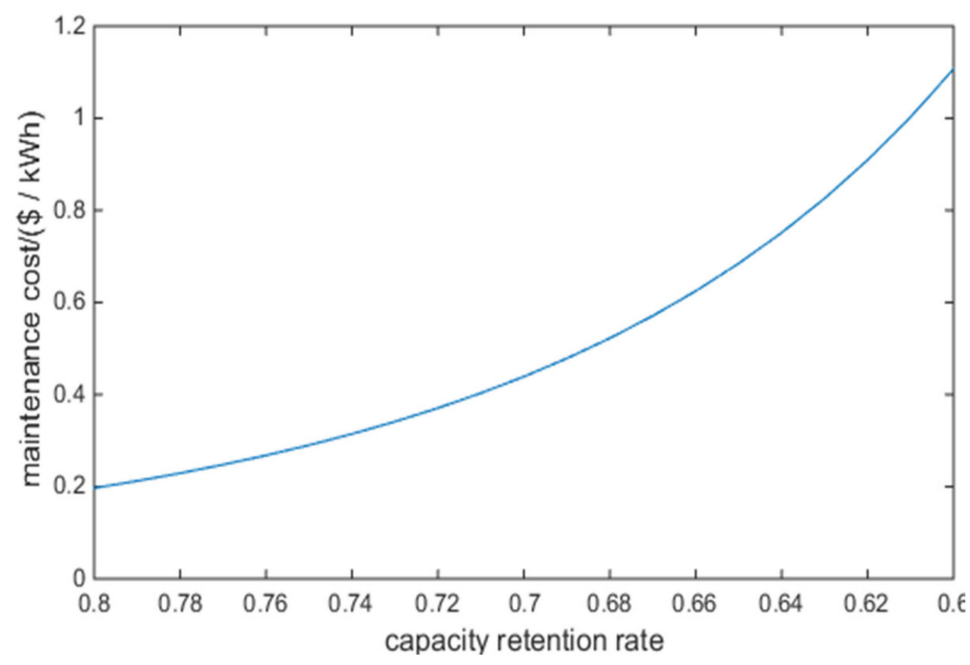

Figure 15. Maintenance cost analysis. 


\section{Conclusions}

The application of energy storage in the power grid is becoming increasingly widespread, and the investment cost of new energy storage devices is higher. In view of this problem, this paper proposes an energy storage device that is composed of retired power batteries from electric vehicles, which is used in the power grid to improve its operational flexibility. Therefore, this paper first analyzes the relationship between the capacity retention rate of electric vehicle power batteries and the number of cycles, and then proposes a model for calculating the capacity retention rate of electric batteries based on the total mileage of electric vehicles. On the one hand, the bounds of the SUESS capacity are updated in order to prevent overcharging or discharging and, on the other hand, the energy storage maintenance costs are updated, which makes the scheduling plan more accurate. When the capacity retention rate drops to $80 \%$, retired power battery constitutes an energy storage device for the power grid. Finally, an energy storage system that was composed of power batteries was used in the distribution network with photovoltaic power plants, and a multi-target day-ahead scheduling model for distribution network operation was established.

From the simulation results, the maximum PV hosting capacity is analyzed in the modified IEEE 37 test system, which verifies the rationality of the second-use energy storage batteries that are participating in the optimal dispatching of the distribution network, and it proves the residual value of the retired batteries. After the SUESS installation, the voltage deviation level of the node is reduced. What is more, it compares the network loss with and without the power four-quadrant grid-connected operation strategy, and proves its advantages. The SUESS output of the four-quadrant power can be regulated by reactive power to avoid unnecessary power loss. Finally, the SUESS is more economical than brand new energy storage.

Author Contributions: The individual contributions of the authors are specified as follows: Conceptualization, L.T. and J.Z (Jian Zhao); methodology, L.T.; software, H.J.; validation, L.T., J.Z.(Jian Zhao) and H.J.; formal analysis, H.J.; writing —original draft preparation, Q.Z. and H.J.; writing—review and editing, J.Z.(Jingjing Zhao) and H.J.; supervision, J.Z.(Jinhui Zhou). All authors have read and agreed to the published version of the manuscript.

Funding: This work was supported by Science and Technology Project of State Grid Zhejiang Electric Power Co., LTD. (5211DS18002S); National Key R\&D Program of China (2018YFB0905105); and National Natural Science Foundation of China (51907114).

Conflicts of Interest: The authors declare no conflict of interest.

\section{References}

1. Zhu, Z.; Zhao, J.; Wei, W. Optimal charging and discharging scheme of battery energy storage system in active distribution network. Autom. Electr. Power Syst. 2016, 40, 47-53.

2. Noh, S.G.; Choi, W.Y.; Kook, K.S. Operating-Condition-Based Voltage Control Algorithm of Distributed Energy Storage Systems in Variable Energy Resource Integrated Distribution System. Electronics 2020, 9, 211. [CrossRef]

3. Zakeri, B.; Syri, S. Electrical energy storage systems: A comparative life cycle cost analysis. Renew. Sustain. Energy Rev. 2015, 42, 569-596. [CrossRef]

4. Li, T.; Shi, S.; Jia, Z. A statistical model for charging power demand of electric vehicles. Power Syst. Technol. 2010, 34, 126-130.

5. Wang, Z.; Li, J. Application of battery cascade utilization device in EV battery charging and swapping station. Electr. Autom. 2012, 34, 49-50.

6. Ochoa, L.F.; Dent, C.J.; Harrison, G.P. Distribution network capacity assessment: Variable DG and active networks. IEEE Trans. Power Syst. 2010, 1, 87-95. [CrossRef]

7. Hashemi, S.; Ostergaard, J. Efficient control of energy storage for increasing the PV hosting capacity of LV grids. IEEE Trans. Smart Grid 2017, 9, 2295-2303.

8. Zhao, J.; Wan, C.; Xu, Z. Spinning Reserve Requirement Optimization Considering Integration of Plug-In Electric Vehicles. IEEE Trans. Smart Grid 2016, 8, 2009-2021. [CrossRef] 
9. Zhao, J.; Wan, C.; Xu, Z. Risk-Based Day-Ahead Scheduling of Electric Vehicle Aggregator Using Information Gap Decision Theory. IEEE Trans. Smart Grid 2015, 8, 1609-1618. [CrossRef]

10. Meng, X.; Sun, F.; Lin, C. Cycle life prediction of power battery. Chin. J. Power Sources 2009, 33, 955-969.

11. Peng, R.; Pedram, M. An analytical mode for predicting the remaining battery capacity of lithium-ion batteries. IEEE Trans. Very Large Scale Integr. (VLSI) Syst. 2006, 14, 441-451. [CrossRef]

12. Liu, N.; Tang, X.; Duan, S. Capacity optimization method for PV-based battery swapping stations considering second-use of electric vehicle batteries. Proc. CSEE 2013, 33, 34-44.

13. Wu, S.; Lu, Y. Research on the Electric Vehicles and Energy Storage Optimal Control in Microgrid and Battery Second Use. Master's Thesis, North China Electric Power University, Beijing, China, June 2017.

14. Awad, A.; Salama, M. Optimal ESS Allocation for Benefit Maximization in Distribution Networks. IEEE Trans. Smart Grid 2015, 8, 1668-1678. [CrossRef]

15. Liu, X.; Aichhorn, A.; Li, H. Coordinated Control of Distributed Energy Storage System with Tap Changer Transformers for Voltage Rise Mitigation under High Photovoltaic Penetration. IEEE Trans. Smart Grid 2012, 3, 897-906. [CrossRef]

16. Koeppel, G.; Geidl, M.; Andersson, G. Value of storage devices in congestion constrained distribution networks. In Proceedings of the International Conference on Power System Technology, Singapore, 21-24 November 2004.

17. Taylor, M.J.; Alexander, A. Evaluation of the impact of plug-in electric vehicle loading on distribution system operations. In Proceedings of the IEEE Power \& Energy Society General Meeting, Calgary, AB, Canada, 26-30 July 2009; pp. 1-6.

18. Vyas, A.; Santini, D. Use of National Surveys for Estimating Full PHEV Potential for Oil Use Reduction. Available online: http://www.transportation.anl.gov/pdfs/HV/525.pdf. (accessed on 20 December 2019).

19. Debnath, U.K.; Ahmad, I.; Habibi, D. Quantifying economic benefits of second life batteries of gridable vehicles in the smart grid. Int. J. Electr. Power Energy Syst. 2014, 63, 577-587. [CrossRef]

20. Li, J.L.; Xiu, X.Q.; Liu, D.T.; Hui, D. Research on the ladder application of decommissioned power battery energy storage system considering policy incentives. High Volt. Eng. 2015, 41,1-7.

21. Zhao, J.; Wang, J.; Xu, Z. Distribution Network Electric Vehicle Hosting Capacity Maximization: A Chargeable Region Optimization Model. IEEE Trans. Power Syst. 2017, 32, 4119-4130. [CrossRef]

22. Zhou, D.; Ren, Z.; Huang, H. Optimal dispatching model of active distribution network considering uncertainty of demand response. Energy Storage Sci. Technol. 2018, 4, 637-644.

23. Zhu, L.; Zhou, X.; Zhang, X. Integrated resources planning in microgrids considering interruptible loads and shiftable loads. J. Mod. Power Syst. Clean Energy 2018, 6, 1-14. [CrossRef]

24. Basmadjian, R. Optimized Charging of PV-Batteries for Households Using Real-Time Pricing Scheme: A Model and Heuristics-Based Implementation. Electronics 2020, 9, 113. [CrossRef]

25. Haghighat, H.; Zeng, B. Stochastic and chance-constrained conic distribution system expansion planning using bi-linear benders decomposition. IEEE Trans. Power Syst. 2017, 33, 2696-2705. [CrossRef]

26. Heitsch, H.; Römisch, W. Scenario reduction algorithms in stochastic programming. Comput. Optim. Appl. 2003, 24, 187-206. [CrossRef]

27. Kersting, W. Radial distribution test feeders. IEEE Trans. Power Syst. 1991, 6, 975-985. [CrossRef]

(C) 2020 by the authors. Licensee MDPI, Basel, Switzerland. This article is an open access article distributed under the terms and conditions of the Creative Commons Attribution (CC BY) license (http://creativecommons.org/licenses/by/4.0/). 